Revue des patrimoines

28 | 2016

Le moulage. Pratiques historiques et regards contemporains

\title{
La création d'une collection nationale de moulages en Belgique. Du musée des Plâtres à la section d'Art monumental des Musées royaux des arts décoratifs et industriels à Bruxelles
}

The creation of a national collection of plaster casts in Belgium. From the "musée des Plâtres" to the section of monumental Art of the Royal museums of Decorative and Industrial Arts in Brussels

\section{Valérie Montens}

\section{OpenEdition}

Journals

Édition électronique

URL : http://journals.openedition.org/insitu/12564

DOI : 10.4000/insitu. 12564

ISSN : $1630-7305$

Éditeur

Ministère de la culture

Référence électronique

Valérie Montens, «La création d'une collection nationale de moulages en Belgique. Du musée des

Plâtres à la section d'Art monumental des Musées royaux des arts décoratifs et industriels à Bruxelles », In Situ [En ligne], 28 | 2016, mis en ligne le 16 mars 2016, consulté le 01 mai 2019. URL : http:// journals.openedition.org/insitu/12564 ; DOI : 10.4000/insitu. 12564

Ce document a été généré automatiquement le 1 mai 2019.

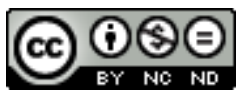

In Situ Revues des patrimoines est mis à disposition selon les termes de la licence Creative Commons Attribution - Pas d'Utilisation Commerciale - Pas de Modification 4.0 International. 


\title{
La création d'une collection
} nationale de moulages en Belgique. Du musée des Plâtres à la section d'Art monumental des Musées royaux des arts décoratifs et industriels à Bruxelles

\author{
The creation of a national collection of plaster casts in Belgium. From the \\ "musée des Plâtres" to the section of monumental Art of the Royal museums of \\ Decorative and Industrial Arts in Brussels
}

Valérie Montens

1 En Belgique comme dans toute l'Europe, les moulages ont connu un véritable âge d'or au $\mathrm{XIX}^{\mathrm{e}}$ siècle. Les collections de reproductions d'œuvres d'art en plâtre se sont développées dans les académies des beaux-arts où l'enseignement du dessin était basé sur la copie des modèles gréco-romains ${ }^{1}$. Par la suite, elles sont apparues dans certaines universités ${ }^{2}$ et dans plusieurs musées et se sont étendues à l'art médiéval et renaissant.

2 À Bruxelles, plus particulièrement, une galerie (nationale) des plâtres antiques a été créée au sein du musée royal de Peinture et de Sculpture (futur musée des Beaux-Arts) dès la fin des années 1840. À dater de cette époque, les acquisitions (et projets d'acquisitions) de reproductions en plâtre se sont multipliées, donnant naissance au musée des Plâtres, en 1863, au musée des Échanges internationaux, en 1884, et finalement à une section d'Art monumental au sein des musées royaux des Arts décoratifs et industriels (ancêtre des musées royaux d'Art et d'Histoire), créés en $1889^{3}$.

3 C'est sur cette histoire que nous voudrions revenir ${ }^{4}$ ici, pour en souligner les moments clés, pour éclairer mieux le rôle de ses acteurs et, enfin, pour comprendre la place 
accordée à cette collection dans la politique culturelle belge de la seconde moitié du XIX siècle.

\section{Le « musée des plâtres »(1849-1889) ${ }^{5}$}

4 Si le musée du Département de Dyle, présentait déjà, au tout début du XIX ${ }^{\mathrm{e}}$ siècle, dans ses salles du palais de l'Ancienne Cour de Bruxelles (actuel palais de Charles de Lorraine), deux "plâtres" antiques, à savoir la Vénus de Médicis et la Vénus du Capitole 6 , il fallut attendre 1849 pour que son héritier, le musée royal de Peinture et de Sculpture, dispose d'une véritable galerie de «moulages d'après des originaux existants dans d'autres collections".

5 Cette année-là, le musée reçut, par l'intermédiaire du consul belge à Athènes, 184 pièces, parmi lesquelles des moulages de reliefs du Parthénon, de l'Erechteion, du temple d'Athéna Nikè, des Propylées, du Théséion et du monument de Lysicrate. Cet ensemble venait s'ajouter à la centaine de moulages qui avaient été déposés, dès 1839, dans la chapelle gothique faisant partie du musée de Sculpture. L'État belge avait acquis ces reproductions de sculptures gréco-romaines et de quelques œuvres de la Renaissance auprès de l'atelier du Louvre pour l'École de gravure de Bruxelles, qui avait été annexée à l'Académie de Bruxelles.

6 Ainsi, en 1849, une "galerie de plâtres » présentant déjà, pour les peintres et les sculpteurs, « de nombreux et d'utiles sujets d'étude ${ }^{7}$ ", fut installée dans les mansardes de l'actuel palais de Charles de Lorraine. Son organisation fut confiée à Eugène Simonis, sculpteur renommé, revenu d'Italie, qui avait été nommé membre de la commission administrative du musée en $1846^{8}$, aidé par le mouleur Jean-Pierre Van den Broeck.

7 Sous son égide, les musées acquirent de nouveaux moulages. En 1850, tout d'abord, ceux que le professeur prussien Zahn avait moulés en Italie, puis en 1852, 16 moulages réalisés par Micheli, mouleur des musées nationaux à Paris (fig. 1). 
Figure 1

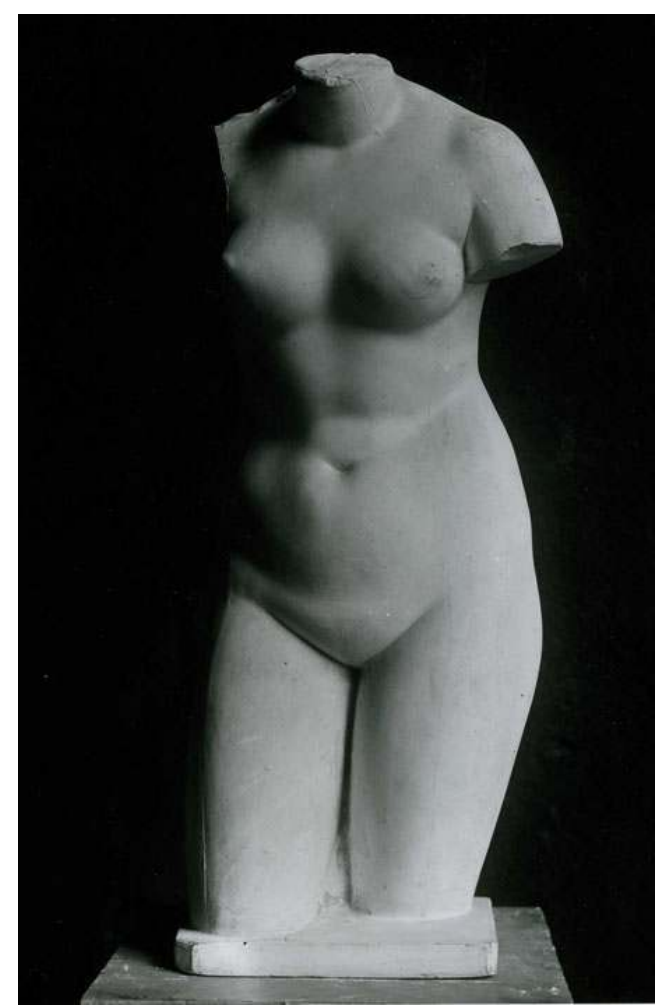

Moulage de la Vénus Médicis acheté en 1852 à Paris.

(c) Musées royaux d'Art et d'Histoire (Institut Royal du Patrimoine Artistique, I.R.P.A.)

8 Par ailleurs, l'atelier de moulage qui avait été mis en place, à l'origine, afin de faciliter la remise en état des plâtres arrivés d'Athènes très abîmés, entama un travail de reproduction d'œuvres de nos régions.

Dans la décennie suivante, une nouvelle impulsion fut donnée à la collection de moulages par le monde politique. En 1860, le ministre Charles Rogier écrivit à la commission du musée :

Dans les principaux musées de l'Europe, et notamment dans ceux d'Allemagne et d'Italie, on a formé des collections aussi complètes que possible et très intéressantes de plâtres d'après les œuvres les plus célèbres de la statuaire antique et de la sculpture moderne. La galerie de sculpture du musée de l'état présente sous ce rapport des lacunes qu'il serait utile de remplir9.

Désormais, il ne s'agissait plus seulement d'offrir des modèles aux artistes, mais surtout d'affirmer la position de la jeune nation qu'était la Belgique sur l'échiquier européen.

11 En réponse à cette demande, le musée proposa de «recueillir en premier lieu tous les ouvrages remarquables que nous trouverons à Paris, et de faire ensuite une tournée rapide en Allemagne et en Italie, afin de compléter notre galerie et lui donner toute l'importance d'un musée de sculpture $»^{10}$.

12 Selon la commission, en effet, "presque tous les beaux modèles de la sculpture antique ont été moulés ou surmoulés à Paris, où il est possible de se les procurer à des conditions beaucoup plus avantageuses, indépendamment du transport qui sera également moins coûteux $»^{11}$. 

1861 et appel fut fait à la commission royale des Monuments ${ }^{12}$ pour donner son avis sur l'utilité de "créer dans les musées de sculpture une section du moyen-âge et de la Renaissance, dans laquelle seraient classés les modèles originaux ou les copies en plâtre de ce que ces époques ont produit de mieux en statues, bas-reliefs, ornements, tombeaux, etc. ». Cette dernière envoya en 1863 une liste comprenant tant des œuvres à mouler dans le pays que des «spécimens remarquables de l'école française et allemande à cause de l'influence qu'elles ont exercée sur nos $\operatorname{artistes}^{13} \%$.

Entretemps, en raison de la pénurie de locaux au musée, le ministre décida de faire transférer la collection des moulages au palais ducal (le futur palais des Académies). Les premières œuvres y furent placées en février 1863 mais l'ouverture au public n'eut lieu qu'en 1867, ainsi qu'en témoigne, dans les archives des musées, un projet de communiqué de presse :

La galerie des plâtres antiques et de la Renaissance installée récemment dans les salles du rez-de-chaussée du Palais ducal, est ouverte au public tous les jours depuis 10 heures du matin jusqu'à 4 heures de relevée. Des cartes d'études seront délivrées aux artistes qui en feront la demande à la commission administrative du musée ${ }^{14}$ (fig. 2).

Figure 2

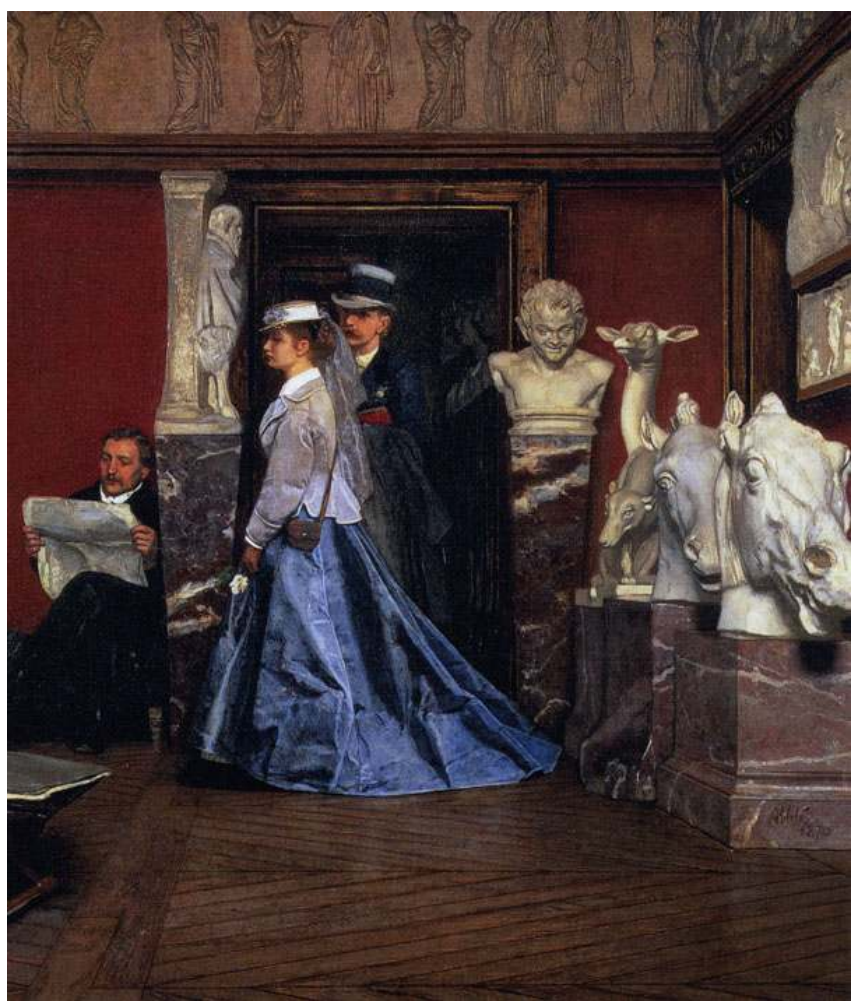

Une salle du musée des Plâtres au palais ducal vue par le peintre hollandais Auguste Allebe, Amsterdam, Stedelijk Museum (d'après M. VAN KALCK. Les Musées royaux des Beaux-Arts de Belgique, vol. 1, 2003, p. 263)

Les achats reprirent donc et, au cours des années qui suivirent, plusieurs importantes séries de moulages entrèrent dans la collection. Citons plus particulièrement 57 œuvres commandées en 1864 à l'atelier du Louvre, les moulages acquis en 1865 à la vente publique de la collection de moulages De Sachy, mouleur de l'École des beaux-arts de 
Paris, ceux achetés en 1867 à Detrémont, mouleur de l'Académie royale des beaux-arts, sans oublier les œuvres fournies par la Société pour le moulage et la reproduction d'objets d'art religieux à Louvain, ainsi que des moulages pris par le sculpteur Emmanuel Colinet sur des monuments et objets $\mathrm{du} \mathrm{XI}^{\mathrm{e}}$ au XVI $\mathrm{X}^{\mathrm{e}}$ siècles.

En 1882, la description des salles ou «cabinets» de ce qu'on appelait désormais le " musée des Plâtres » parut sous la plume de Jean-Baptiste Rousseau (1829-1891). Peintre, écrivain et critique d'art, ce dernier était depuis 1865 secrétaire de la commission des Monuments. Déjà auteur, en 1868, d'un important rapport sur la pratique de l'enseignement, il avait été nommé, en 1877, inspecteur des Beaux-Arts ${ }^{15}$.

On ne sera donc pas surpris de lire, dans son introduction, un plaidoyer en faveur de ce type de collection :

Un musée ne doit pas seulement être une collection de raretés : ainsi compris, ce ne serait plus qu'un monument de luxe et de vanités puérils. Il est fait avant tout pour donner des leçons, pour façonner le goût de la foule, qui ne hante pas les Académies, pour parfaire l'éducation de l'artiste en le mettant en présence des chefs-d'œuvre de son $\operatorname{art}^{16}$.

Sa description rend bien compte de la situation de cette collection à ce moment :

Après les quinze salles (de moulages antiques) que nous venons de visiter, on en parcourt encore trois ou quatre, où le Moyen-Âge, la Renaissance, l'art moderne sont représentés à leur tour, mais d'une façon, cela va de soi, très sommaire. Pour ces différences époques, le Musée des Plâtres est encore à faire, ou plutôt c'est dans une autre collection qu'il faut aller les étudier, celle qui a été formée à l'aide des Échanges internationaux ${ }^{17}$.

\section{Le « musée des Échanges » (1871-1884)}

En 1867, à l'initiative du premier directeur du musée de South Kensington de Londres, un pavillon modèle fut installé à l'Exposition universelle de Paris, afin de faire la démonstration de ce que devait être un musée des moulages. À cette occasion, des princes de plusieurs maisons régnantes d'Europe se réunirent à l'invitation du prince de Galles, pour favoriser des échanges de reproductions.

Notre pays, représenté par le comte de Flandre, participa à la signature de la « convention pour l'encouragement universel des reproductions d'œuvres d'art dans l'intérêt de tous les pays " qui jetait les bases d'une union internationale permettant à chaque pays, représenté par une commission particulière, de faire connaître les reproductions qu'il pouvait fournir et d'obtenir les reproductions nécessaires pour ses musées.

Dans ce contexte, la Belgique mit sur pied, à partir de 1871, la Commission royale belge des échanges internationaux. Au sein de celle-ci, une section artistique fut constituée et dotée d'un comité présidé par Édouard Fétis, conservateur du musée royal de Peinture et de Sculpture. Parmi ses membres figuraient un architecte, Alphonse Balat, un sculpteur, Guillaume Geefs, un peintre, Charles Debrou, ainsi que deux professeurs d'archéologie, le chanoine Delvigne et l'abbé Reusen. Mais sa cheville ouvrière était son secrétaire, Adolphe van Soust de Borkenfeld, directeur au département des Beaux-Arts.

La section artistique entama rapidement la reproduction par moulage de monuments anciens de notre patrimoine national. Il s'agissait, entre autres, de la cheminée du Franc de Bruges ( $\mathrm{xVI}^{\mathrm{e}}$ siècle), des sculptures des boiseries de l'église Saint-Paul à Anvers (XVII siècle), de trois petits monuments votifs de l'église Saint-Pierre à Louvain, du mausolée 
Ferry de Gros à Bruges, des fonts baptismaux des églises de Saint-Barthélémy à Liège et de Saint-Martin à Hal, etc.

Réalisés dans les sous-sols du palais ducal, dans l'atelier qui y avait été aménagé pour Van den Broeck, les moulages furent placés dans une salle de ce bâtiment, complétant la collection du musée des Plâtres.

Mais en même temps que s'exécutaient les premiers moulages du fonds belge, se formait un noyau de reproductions étrangères. Le musée de South Kensington fut un des premiers fournisseurs de la section artistique. Il envoya à Bruxelles les moulages des chaires du dôme et du baptistère de Pise (XIII ${ }^{\mathrm{e}}$ et $\mathrm{XIV}^{\mathrm{e}}$ siècles) et de la chasse de SaintSebald à Nuremberg, en échange de la reproduction de la cheminée du Franc de Bruges (fig. 3, fig. 4).

Figure 3

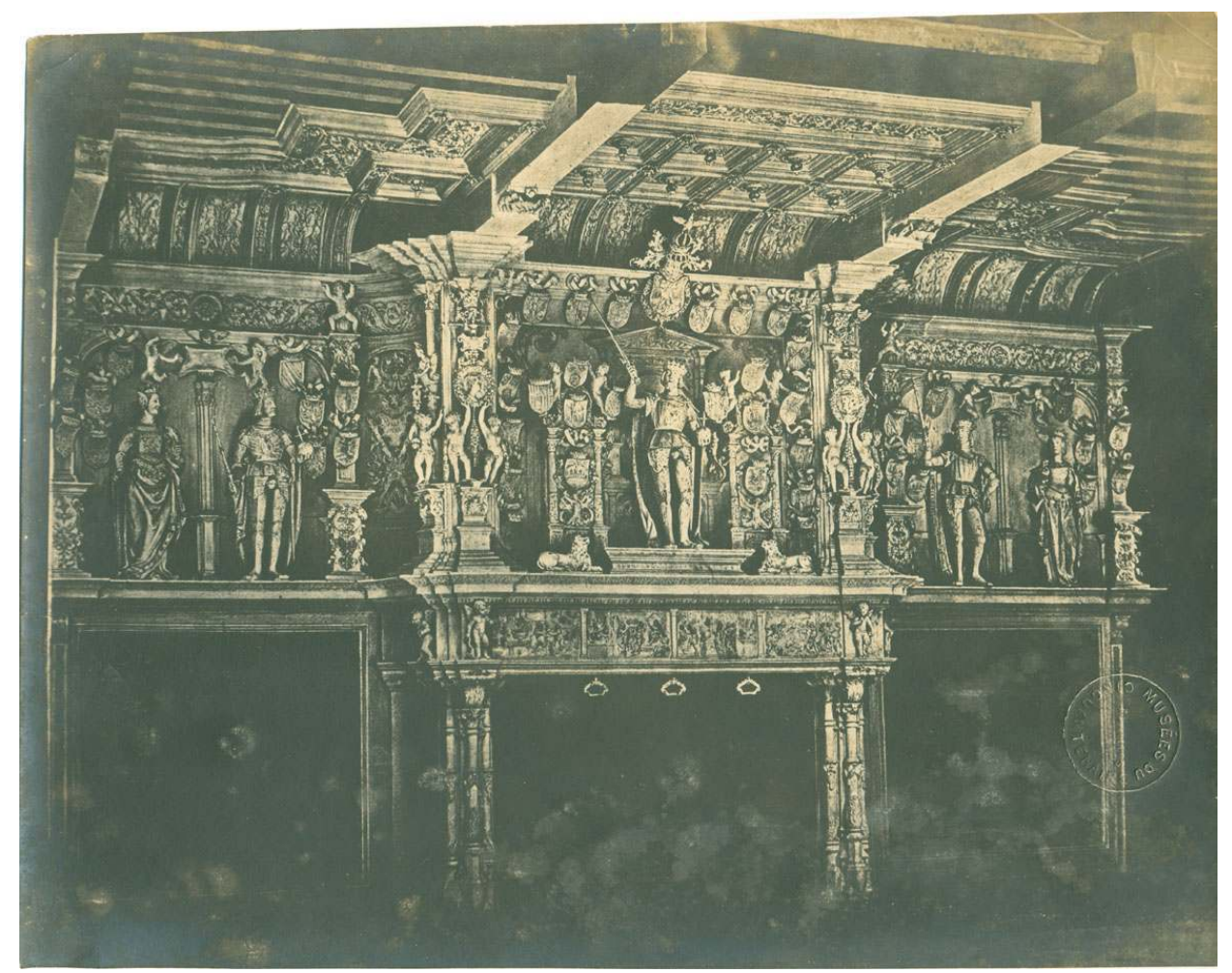

Cheminée de Lancelot Blondeel, $\left.x \mathrm{~V}\right|^{\mathrm{e}}$ siècle, Franc de Bruxelles. Archives MRAH.

(c) Musées royaux d'Art et d'Histoire. 


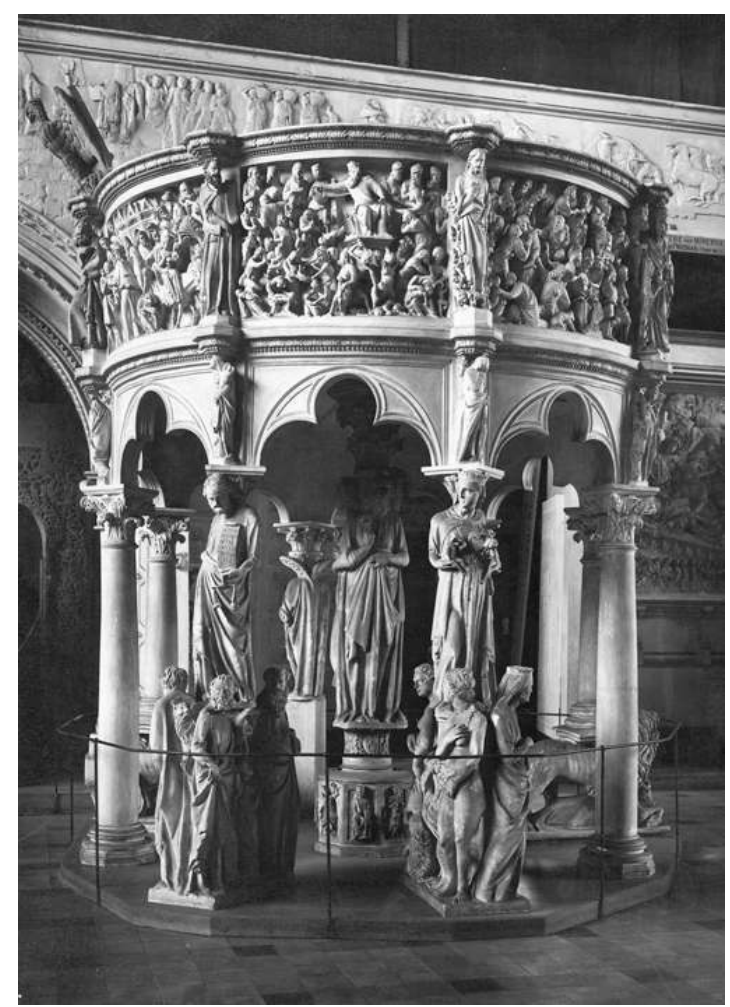

Chaire de Nicola Pisano, XIII' siècle, baptistère de Pise.

(c) I.R.P.A.

Contact fut également pris avec les musées allemands de Nuremberg et de Dresde qui fournirent l'un des reproductions de pierres tombales provenant de Naumbourg et d'Hildesheim, et l'autre le moulage de deux bases de candélabres conservées à l'Albertinum.

Les moulages provenant des échanges étaient installés dans l'ancienne salle de la Société philharmonique, rue de l'Évêque. Mais au début des années 1880, l'État, qui devait procéder à la démolition de ces bâtiments pour la construction de l'hôtel des postes et télégraphes, demanda le transfert de ces reproductions d'œuvres d'art dans un des bâtiments construits pour l'Exposition de 1880 sur l'ancien champ des manœuvres (l'actuel parc du Cinquantenaire).

Lors de l'exposition organisée à l'occasion du cinquantenaire de l'indépendance nationale, la cheminée monumentale du Franc de Bruges et le tabernacle de l'église de Léau avaient déjà été érigés dans le "pavillon de l'art ancien» au palais du Cinquantenaire. Sans aucun doute Jean-Baptiste Rousseau, secrétaire de la commission des fêtes et ordonnateur des principales manifestations officielles dont la capitale fut alors le théâtre, n'y était-il pas étranger.

En tout cas, c'est lui qui dirigea les travaux d'aménagement (chauffage, plafonnage, etc.) exécutés après 1880 dans le pavillon nord du palais pour y installer la collection des plâtres de la commission royale belge des Échanges internationaux, avec Alphonse Balat, président du comité de la section artistique. Le déménagement commença en 1883.

Tout ceci fut officialisé en 1884 : 
Le fonds des reproductions d'œuvres artistiques, réuni par les soins de la Commission royale belge des échanges internationaux, constituera un établissement distinct administré par ladite commission, sous la dénomination de Musée des échanges internationaux, collection de moulages et reproductions de monuments et objets d'art ${ }^{18}$.

\section{La section d'Art monumental des musées des Arts décoratifs et industriels (1889-1928)}

En Belgique, le projet de création d'un musée des « arts industriels » destiné à permettre aux futurs ouvriers de recevoir un enseignement équilibré entre technique et sensibilisation artistique naquit dans la seconde moitié $d u x x^{e}$ siècle, à la suite de l'Exposition de Londres, en 1851. Les critiques relatives à la qualité esthétique des produits présentés dans la capitale britannique avaient en effet débouché sur un élan de réformes de la conception des disciplines décoratives et de l'organisation de l'enseignement artistique ${ }^{19}$.

Il fallut toutefois attendre 1880 et les deux expositions consacrées, dans le cadre de ce jubilé, à la présentation des disciplines décoratives (l'une sur les arts industriels contemporains, l'autre sur les arts décoratifs anciens) pour qu'un pas décisif fût accompli vers sa réalisation. Celles-ci permirent en effet d'expérimenter, à échelle réduite, l'éventuelle attribution d'une partie des locaux à un musée spécialisé.

En 1884, la commission administrative du musée de Peinture et de Sculpture reçut de la part de son ministre de tutelle une demande de mise en dépôt, au musée des Échanges, d'une partie des moulages conservés au palais des Académies :

Il y a dans une des salles du palais des Académies qui ne peut être éclairée qu'au gaz et qui est habituellement fermée au public, une série de modèles d'architecture et d'art appliqué actuellement perdus pour l'étude et qui trouveraient une meilleure place au musée des arts décoratifs actuellement en voie de formation au champ des manœuvres ${ }^{20}$.

Un premier envoi eut donc lieu en février 1884.

À juste titre, le ministre proposa également d'examiner s'il ne conviendrait pas, en général, que tous les moulages ayant un caractère d'art appliqué à l'industrie tels que coupes, candélabres, etc. fissent dorénavant partie du musée des Arts décoratifs qui, de son côté, pourrait céder au musée royal de Sculpture les "moulages d'art pur » qu'il possède, tels que les sculptures provenant des fouilles d'Olympie, le Mercure de Praxitèle, la Vierge de Michel Ange. "Il importe, semble-t-il, dans l'intérêt même des études artistiques, que le caractère de chaque musée soit nettement déterminé et ne donne lieu à aucune confusion », soulignait-il alors ${ }^{21}$.

Mais en 1889, c'est l'ensemble du musée des Plâtres qui fut transféré au Cinquantenaire où l'État avait décidé d'installer la collection des «antiquités " (art ancien) du musée royal d'Armes, Armures et Antiquités précédemment conservée à la porte de Hal, afin d'y créer le nouvel ensemble des « musées royaux des Arts décoratifs et industriels ${ }^{22}$ ».

Le musée des Plâtres forma ainsi la section de "l'Art monumental " et fut confiée à Antoine Van Hammée, artiste peintre et professeur à l'Académie des beaux-arts de Bruxelles. Désormais réunies au Cinquantenaire, les deux collections de reproductions, le musée des Plâtres et le musée des Échanges, restèrent toutefois administrativement distinctes (fig. 5). 
Figure 5

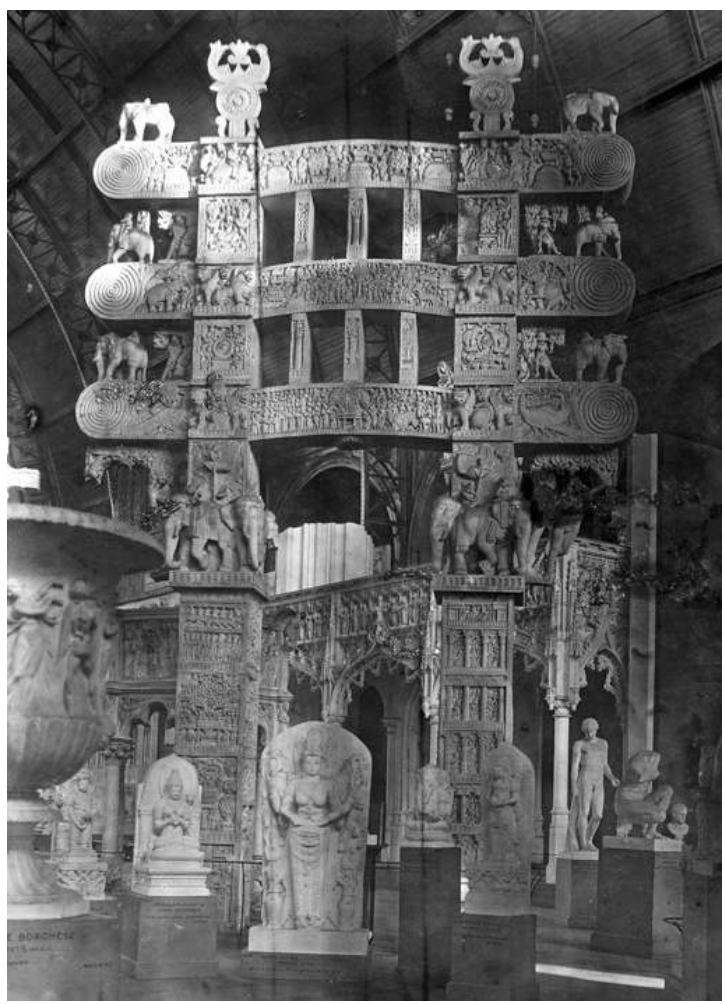

Vue de la section d'Art monumental vers 1900.

(c) I.R.P.A.

Le musée des Échanges fut confié à la gestion d'Henry Rousseau, fils du directeur des Beaux-Arts, qui fut nommé, en 1893, secrétaire du comité de la section artistique de la commission royale belge des Échanges internationaux.

Cette organisation permit au musée de compléter sa collection de reproductions d'œuvres anciennes. Toute proposition d'acquisition d'un moulage, par voie d'échange ou d'achat, ou de moulage d'une œuvre d'art de nos régions était donc soumise au comité de la section artistique qui en évaluait l'intérêt. Ce dernier intervenait également dans le placement des moulages dans les salles des musées.

$\mathrm{Au}$ début des années 1890, en effet, la présentation des moulages méritait des améliorations. Si l'on en croit le parlementaire libéral et bourgmestre de Bruxelles, Charles Buls, les modèles y étaient alors placés «en dépit du bon sens, sans méthode ». L'homme politique regrettait qu'il soit dès lors «impossible de se rendre compte des variations qu'a subies une forme architecturale au cours des siècles ». Or, « les architectes et les artistes décorateurs qui ornent nos monuments sont constamment amenés à emprunter aux arts anciens les éléments d'ornementation ou les formes architecturales qu'ils emploient. Il convient, par conséquent, qu'ils aient sous les yeux des collections de bons modèles $»^{23}$.

Pareille critique se retrouvait encore en 1893, au Sénat, chez le comte Goblet d'Alviella qui compara les salles latérales à une «véritable Babel, où sont confondus tous les âges, tous les pays, tous les ordres, tous les styles ${ }^{24} »$. 
désordre qui régnait dans la section d'Art monumental des musées fut, encore une fois, dénoncé en 1896, au Parlement, cette fois par le futur ministre socialiste des Sciences et des Arts, Jules Destrée, qui livra lui aussi, un rapport détaillé de sa visite :

Dans la grande salle, on trouve d'abord ce joli monument grec connu sous le nom de lanterne de Démosthène, puis une porte de temple hindou, la chaire de Nicolas de Pise et des tombeaux de la Renaissance. On peut excuser ce désordre par la nécessité imposée par les dimensions de ces pièces. Mais dans les petites sections la confusion est inexplicable. Je trouve dans la section égyptienne des figures du fronton du Parthénon, dans la section assyrienne une idole à quatre faces provenant d'Athènes, et nulle mention sur tous ces plâtres ne permet au profane de s'y retrouver ou de savoir ce qu'il représente. L'art grec remplit des salles à droite et à gauche, alors que la logique commande de les réunir: on semble ne pas se douter que l'art grec connut diverses époques et qu'il eut été intéressant de montrer sa filiation avec l'art d'Égypte et d'Orient. Dans l'époque romane, je trouve la reproduction des statues de la cathédrale de Naumbourg, des panneaux arabes, les statuettes de l'école de Bourgogne et l'empereur Julien, du Louvre. Voilà qui doit donner une étrange idée de l'époque romane! Dans une autre section romane, des figures de la cathédrale de Reims! Dans la section Renaissance, un tohu-bohu d'italiens, de français, de flamand, sans distinction et, pour comble, Mme Récamier ! Sur la majorité de ces plâtres, pas d'inscription ni d'indication! Des numéros seulement; et si l'on demande le catalogue, on vous répond qu'il n'y en a pas ${ }^{25}$ ! (fig. 6)

Figure 6

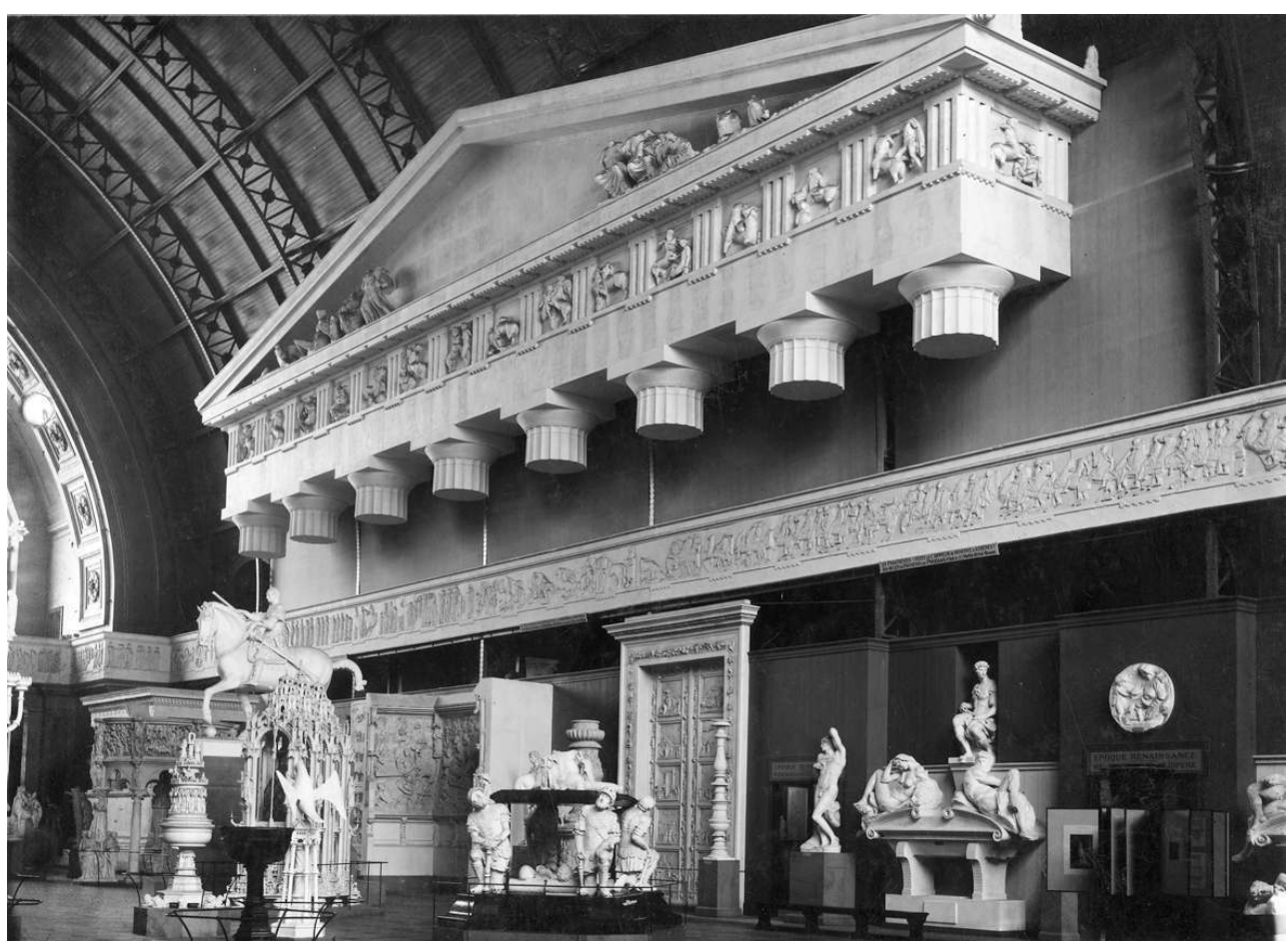

Vue du grand hall de la section d'Art monumental

(c) I.R.P.A.

En 1897, année qui vit le site du Cinquantenaire accueillir une partie de l'Exposition universelle, parurent simultanément un Guide officiel de la section d'art monumental rédigé par Antoine Van Hammée et des Notes illustrées sur l'art monumental et les moulages typiques du musée des échanges d'Henry Rousseau! Plus d'un tiers des 600 moulages répertoriés par 
Van Hammée et Rousseau étaient des reproductions d'œuvres de l'Antiquité classique, offrant un panorama très complet de l'histoire de la sculpture grecque et romaine. À ces œuvres «phares» que sont l'Apollon de la Glyptothèque de Munich, la Victoire de Samothrace (musée du Louvre), la Niké d'Olympie, le Mausolée d'Halicarnasse (British Museum), l'Hercule dit du Belvédère (musée du Vatican), le Génie suppliant (musée de Berlin), l'Hercule Farnèse (musée de Naples), l'Apollon Sauroctone, le Gladiateur Borghèse, la Vénus de Milo et la Vénus Genitrix (musée du Louvre), ou la statue de Septime Sévère de la collection de Somzée, s'ajoutaient des bas-reliefs et des fragments (chapiteaux, corniches, profils de moulures...) destinés à montrer «la suite des transformations des divers éléments de l'architecture » (fig. 7).

Figure 7

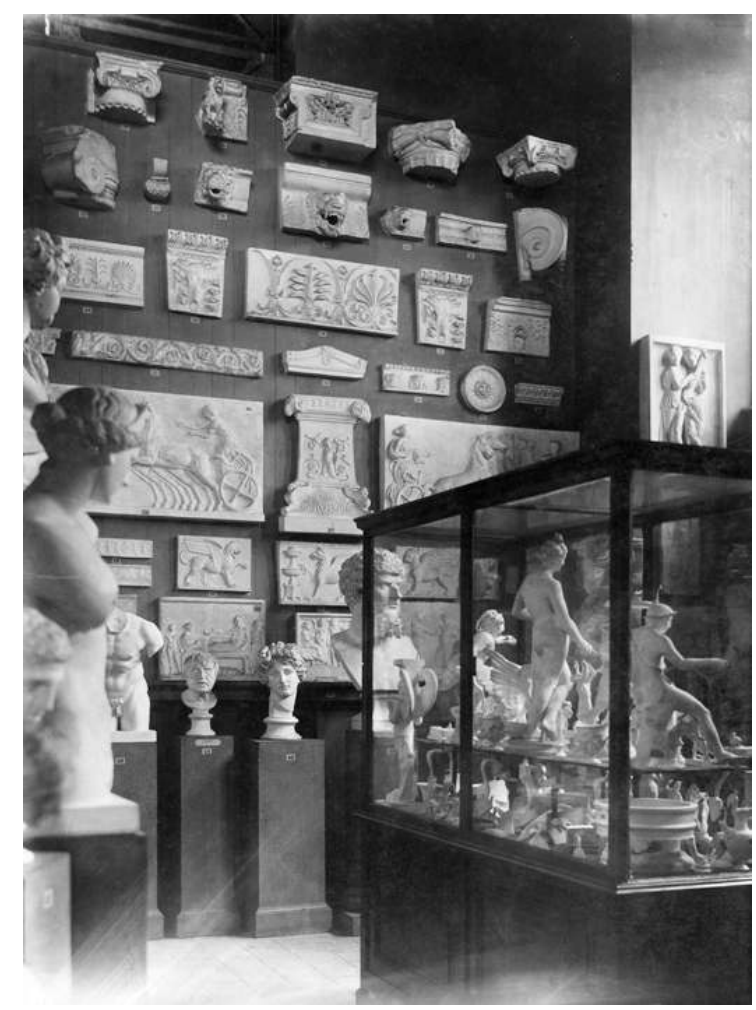

Vue de la salle d'Art gréco-romain.

(c) I.R.P.A. présentées dans la section. Il s'agissait en majorité d'éléments décoratifs de monuments religieux et civils remarquables de Belgique, de France et d'Allemagne, ainsi que de quelques reliquaires, ciboires et objets conservés dans les trésors des églises.

Enfin, la Renaissance était représentée par un tiers des moulages. Tombeaux, médaillons, reliefs, bustes d'origine italienne et française surtout y voisinaient avec des spécimens d'éléments architecturaux tels que cheminées, portes, jubés et grilles de France, d'Italie et des anciens Pays-Bas.

Désormais, la section artistique de la commission royale belge des Échanges internationaux se préoccupait surtout de former des collections de pièces pouvant servir à l'enseignement des métiers d'art, répartis en diverses catégories telles que ferronnerie, dinanderie, menuiserie artistique, ameublement, sculpture décorative monumentale, etc. 
Henry Rousseau voyagea en France, en Angleterre, en Hollande afin de faire des propositions de moulage, d'échange ou d'achat. La collection s'enrichit ainsi de séries de serrures, heurtoirs et ferronneries diverses, de fonts baptismaux, de lutrins et d'aquamaniles, de modèles divers pour orfèvres et ciseleurs, de panneaux, portes, lambris, de bases, chapiteaux, écoinçons et autres détails d'architecture du Moyen Âge jusqu'au XVIII ${ }^{e}$ siècle (fig. 8).

Figure 8

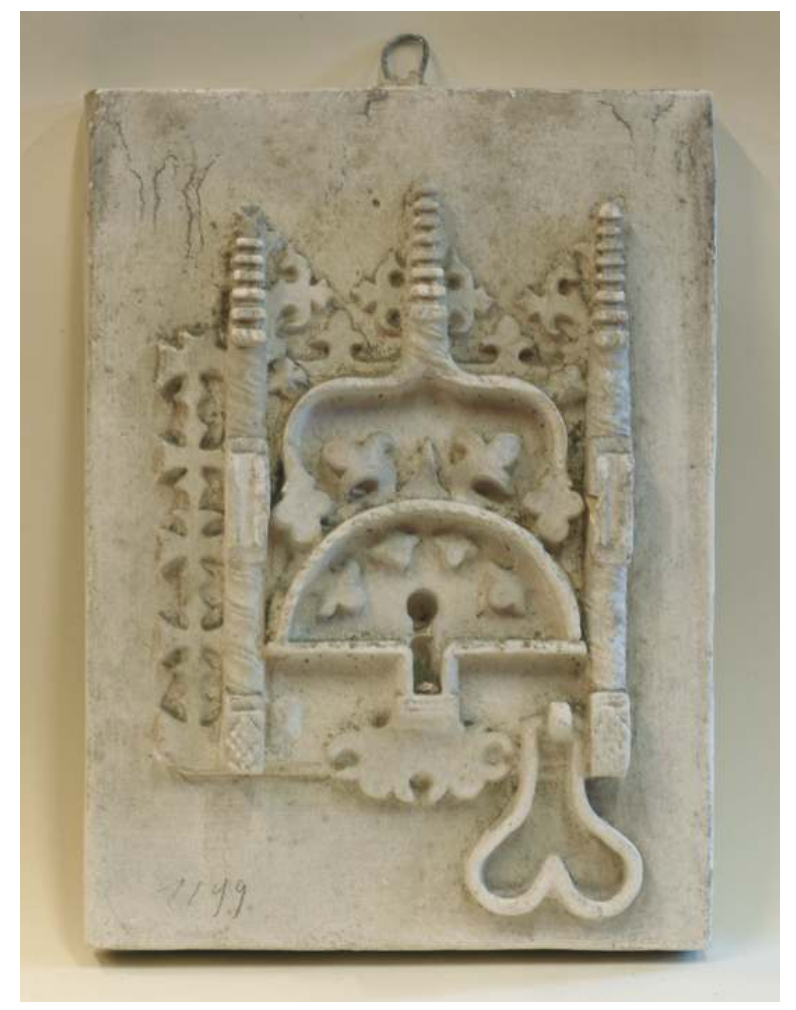

Serrure de meuble, $x v I^{e}$ siècle.

(C) I.R.P.A.

À l'aube $\mathrm{du} \mathrm{xx}^{\mathrm{e}}$ siècle, priorité fut donnée au patrimoine national. Certes, plusieurs œuvres antiques entrèrent encore dans la collection par voie d'échange avec les musées de sculpture allemands de Dresde, Berlin et Munich. Cependant l'attention se porta davantage sur l'illustration de l'évolution de l'histoire de l'art dans nos régions, du Moyen Âge aux Temps modernes. Ainsi Henry Rousseau fut-il chargé de missions d'investigation dans diverses localités du pays d'où il ramena des propositions de moulage d'œuvres « dignes à la fois d'être répandues, données pour modèles, et d'occuper dans les musées étrangers, comme dans le nôtre, une place à laquelle leur donnent droit et leur intérêt et leur beauté26 ${ }^{26}$ (fig. 9). 


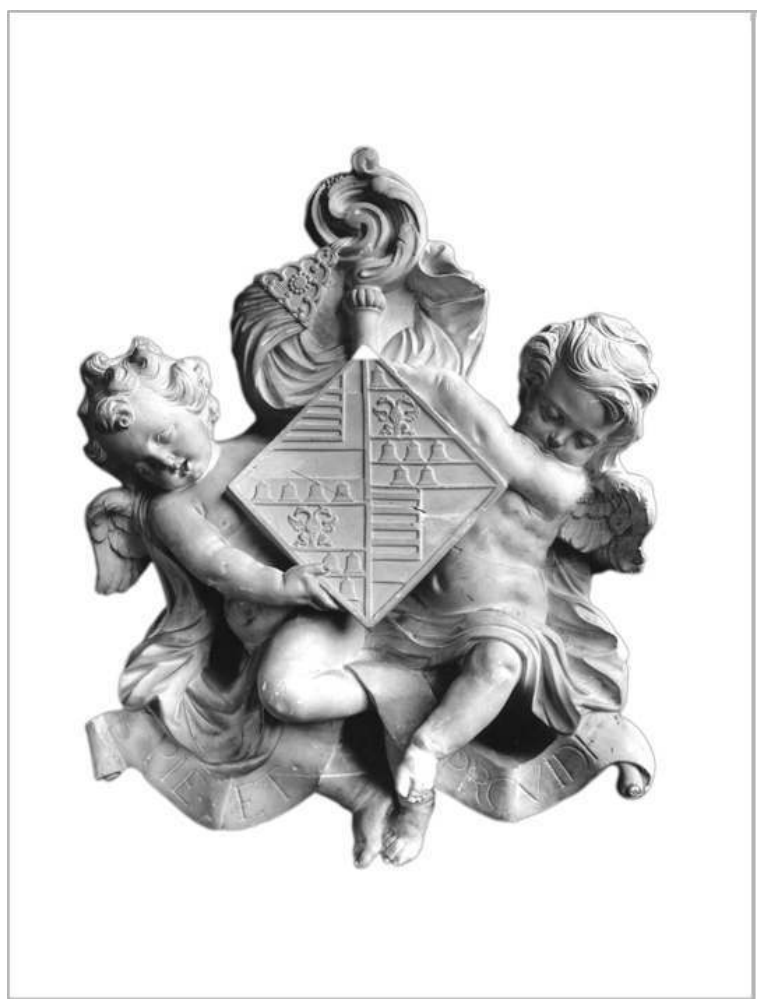

Hasselt, Église des Récollets : détail de tombeau de C. de Lamboy sculpté par A. Quellin le Jeune, XVII siècle.

(c) I.R.P.A.

Dans la même optique, en 1900, deux membres de la section artistique de la commission royale belge des Échanges internationaux suggérèrent de placer aussi dans le musée des maquettes représentant les coupes des monuments historiques du pays, analogues à celles installées dans le musée de Sculpture comparée du Trocadéro ${ }^{27}$.

Si la Belgique ne possède pas, comme la France, des séries de monuments permettant de reconstituer l'enchaînement ininterrompu des évolutions successives de l'art de l'architecture, les reproductions de certains de ses monuments, à petite échelle, et en coupes permettant d'en étudier la structure n'en constitueraient pas moins des documents d'une haute utilité, ",

En 1900, Rousseau publia un nouveau guide du visiteur reprenant cette fois plus de 2000 numéros, soit pratiquement la totalité de la collection. Et cinq ans plus tard, il sortit une nouvelle édition, augmentée de 280 numéros! Parmi ces derniers, il y avait seulement une vingtaine de moulages de pièces gréco-romaines, mais près de 90 moulages d'œuvres médiévales et 140 reproductions illustrant l'art de la Renaissance et des Temps modernes.

51 Mais, à cette époque déjà, une série de plâtres furent relégués dans les sous-sols des musées. Par exemple, la reproduction complète du grand portail de l'église Saint-Servais, à Maastricht, obtenue en 1903 par échange avec le Rijksmuseum d'Amsterdam, n'était pas montée dans le pavillon de l'Art monumental, faute d'espace suffisant. Dès décembre 1907, le comité de la section de l'Art monumental se saisit de la question, 
cherchant à obtenir auprès des autorités de tutelle une extension de ses locaux dans la galerie courbe du musée. Cinq années plus tard, la question ressurgit et des plans furent dressés pour transférer la collection dans de nouveaux locaux, projet contrecarré par la Première Guerre mondiale.

Entre-temps, Henry Rousseau avait entrepris un reclassement de l'ensemble de la collection en 1910, lequel aboutit à la rédaction d'un nouveau catalogue, en 1913. La collection, comprenant pas moins de 5135 numéros, y était subdivisée en quatre périodes chronologiques : l'Antiquité, le Moyen Âge, la Renaissance et les Temps modernes. Avec 2300 numéros, la section médiévale était devenue la plus importante.

Mais, à l'issue de la Première Guerre mondiale, la section artistique de la commission royale belge des Échanges internationaux fut supprimée par un arrêté royal et ses attributions remises au conservateur en chef des musées, désormais appelés musées royaux du Cinquantenaire, et au comité de la $3^{\mathrm{e}}$ section desdits musées.

Et, si le catalogue dressé en 1913 fut réédité en 1926, la section d'Art monumental des musées fut officiellement supprimée en 1928 et son conservateur admis à la retraite.

C'est en effet à cette époque que l'attrait pour les moulages commença à s'affaiblir. Avec le développement des reproductions photographiques, notamment, la vocation pédagogique du musée des Moulages disparut progressivement pour laisser la place à une nouvelle logique commerciale dans laquelle les moulages n'avaient plus d'autre valeur que marchande.

démantèlement de la collection de moulages débuta véritablement au début des années 1930, lorsqu'une partie du bâtiment qui abritait ces reproductions dut être cédée au Musée scolaire et au musée de l'Armée. La plupart des reproductions en plâtre, ainsi que les moules de l'atelier, furent transférés vers l'autre aile du palais du Cinquantenaire, dans les sous-sols du Palais mondial, leur emplacement actuel.

57 Au cours de cette opération, de nombreux moulages ont été endommagés ou détruits. Quelques-uns sont restés en place pendant de nombreuses années, notamment ceux du fronton et la frise du Parthénon. D'autres ont été installés dans les salles d'exposition des musées. Un musée de Moulages antiques, notamment, a été installé dans le local attenant à l'exposition des fouilles d'Apamée mais il fut détruit par l'incendie du 19 février 1946.

Pourtant, en 1930, encore, les musées du Cinquantenaire, désormais appelés musées royaux d'Art et d'Histoire, accueillirent, dans la vaste galerie qui domine leur grand narthex, «l'Exposition internationale des moulages». Sous l'égide de l'office international des musées de l'Institut de coopération intellectuelle de la Société des Nations, celle-ci présenta trois cents moulages environ, rassemblés suivant un ordre chronologique, depuis l'Antiquité égyptienne jusqu'à la fin du XVIII ${ }^{\mathrm{e}}$ siècle (fig. 10). 
Figure 10

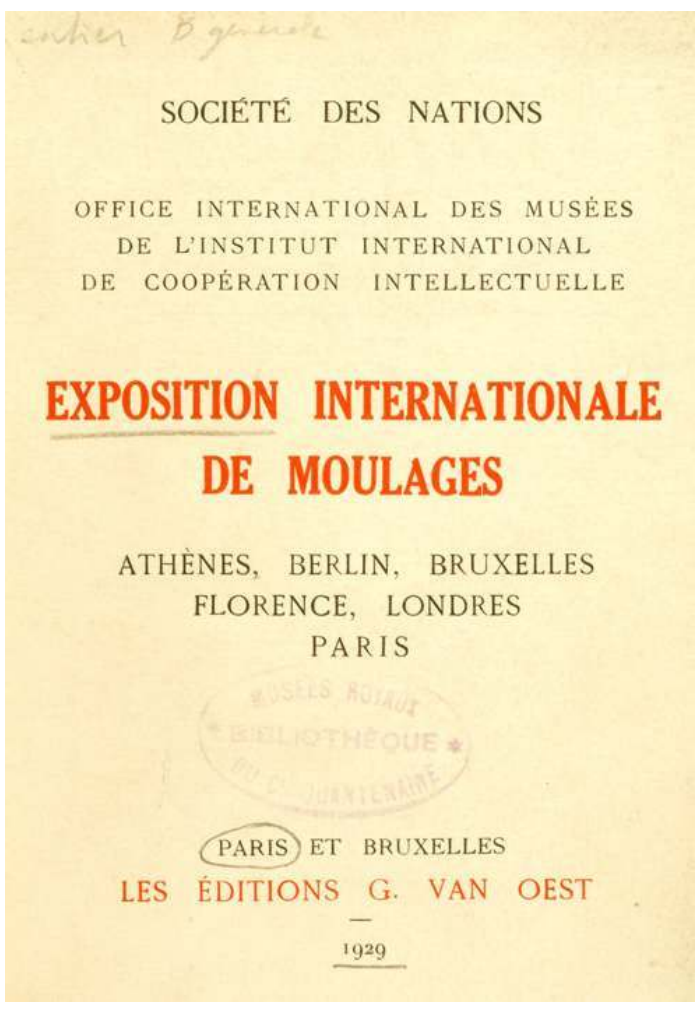

Catalogue de l'Exposition internationale de moulages, Paris et Bruxelles, 1929. Bibliothèque Musées royaux d'Art et d'Histoire.

(c) Musées royaux d'Art et d'Histoire.

\section{Biographie}

Ancienne archiviste, Valérie Montens est docteur en histoire et licenciée en histoire de l'art de l'université libre de Bruxelles. Après une thèse consacrée à l'histoire du palais des Beaux-Arts de Bruxelles, elle a poursuivi ses recherches dans le domaine de l'histoire culturelle, s'attachant plus particulièrement au rôle des hommes d'affaires. En fonction depuis 2000 aux Musées royaux d'Art et d'Histoire où elle a été successivement responsable du service éducatif et du service des archives, elle a publié plusieurs articles sur l'histoire de l'institution et de ses collections.

\section{BIBLIOGRAPHIE}

« Dossier Le moulage ». La vie des musées, AFMB, n¹5, 2000-2001.

Échanges internationaux de reproductions artistiques. Conférence de Bruxelles, 16-19 septembre 1885. Bruxelles : Hayez, 1885.

LOIR, Christophe. « La Belgique et la perception de l'art antique avant 1830 : le développement des collections de plâtres de statues antiques à usage pédagogique ». Dans TSINGARIDA, Athéna, KURTZ, 
Donna (éd.). Appropriating Antiquity. Saisir l'Antique. Collections et collectionneurs d'antiques en Belgique et en Grande-Bretagne au XIX ${ }^{e}$ siècle. Bruxelles : Le Livre Timperman, 2002, p. 33-72.

MONTENS, Valérie. « Aux sources de l'histoire des moulages en plâtre en Belgique : les archives de la section artistique de la Commission royale belge des Échanges internationaux et de l'atelier de moulages des musées royaux d'Art et d'Histoire ». Bulletin des Musées royaux d'Art et d'Histoire, t. 77, 2006, p. 219-240.

MONTENS, Valérie. Les moulages des Musées royaux d'Art et d'Histoire. Histoire de la collection et de l'atelier des reproductions en plâtre. Bruxelles : MRAH, 2008.

« Nouvelles du Patrimoine. Les moulages en plâtre ». Association des Amis de l'UNESCO, n86, avriljuin 2000, p. 8-28.

OFFICE INTERNATIONAL DES MUSÉES DE L'INSTITUT INTERNATIONAL DE COOPÉRATION INTELLECTUELLE. Exposition internationale de moulages. Athènes, Berlin, Bruxelles, Florence, Londres, Paris. Paris/ Bruxelles : éd. G. Van Oest, 1929.

ROUSSEAU, Henry. Catalogue sommaire des moulages. Musées royaux du Cinquantenaire à Bruxelles. $3^{e}$ section. Bruxelles : 固S. Winandy医, 1926.

ROUSSEAU, Henry. Notice sommaire. $3^{e}$ section. Musées royaux du Cinquantenaire à Bruxelles. Aile nord. Bruxelles, octobre 1921.

ROUSSEAU, Henry. Catalogue sommaire des moulages. Musées royaux du Cinquantenaire. Bruxelles, 1913. ROUSSEAU, Henry. Guide illustré du visiteur. Musées royaux des Arts décoratifs et industriels. Section d'art monumental. Bruxelles, $2^{\mathrm{e}}$ éd., 1905.

ROUSSEAU, Henry. Promenade méthodique dans le Musée d'Art monumental. Musées royaux des Arts décoratifs et industriels. Bruxelles : impr. Chevalier, 1902.

ROUSSEAU, Henry. Guide du visiteur. Musées royaux des Arts décoratifs et industriels. Section d'art monumental. Bruxelles, 1900.

ROUSSEAU, Henry. Notes illustrées sur l'art monumental et les moulages typiques du musée des Échanges. Court-Saint-Étienne : Victor Chevalier, 1897.

SKINKEL-TAUPIN, Claire. « L'Atelier de moulages des MRAH ». Bulletin des MRAH, Bruxelles, t. 60, 1989, p. 63-66.

VAN DEN DRIESSCHE, Bernard. «L'antiquité et les moulages en plâtre en Belgique : 1834-1930 ». Dans TSINGARIDA, Athéna et VERBANCK, Annie (éd.). L'Antiquité au service de la modernité ? Réception de l'Antiquité classique en Belgique au XIX ${ }^{e}$ siècle. Actes du Colloque international organisé du 27 au 29 avril 2005 à l'université libre de Bruxelles et au Musée royal de Mariemont. Bruxelles : Le Livre Timperman, 2008, p. 341-353.

VAN DEN DRIESSCHE, Bernard. « Les moulages en Belgique ». Courrier du passant. Bulletin du musée de Louvain-la-Neuve, ${ }^{\circ} 68$, avril-mai 2001, p. 4-24.

VAN DEN DRIESSCHE, Bernard. « Les moulages en plâtre dans les académies et les universités belges ». La vie des musées, $\mathrm{n}^{\circ} 15,2001-2002$, p. 63-66.

VAN HAMMÉE, Antoine. Section d'art monumental. Guide officiel. Musées royaux des arts décoratifs et industriels. Bruxelles, 1897.

VAN KALCK, Michèle. «La collection de moulages ou collection de plâtres d'après l'antique, de l'époque de la Renaissance et du Moyen Âge ». Archiacti(e)ve, MRBAB, n5, mars 2000, p. 3-9. 
VAN KALCK, Michèle (dir.). Les musées royaux des Beaux-Arts de Belgique. Deux siècles d'histoire.

Bruxelles : éditions Racines, 2003.

\section{NOTES}

1. - LOIR, Christophe. «La Belgique et la perception de l'art antique avant 1830 : le développement des collections de plâtres de statues antiques à usage pédagogique ». Dans TSINGARIDA, Athéna (éd.). Appropriating Antiquity. Saisir l'Antique. Collections et collectionneurs d'antiques en Belgique et en Grande-Bretagne au XIX siècle. Bruxelles : Le Livre Timperman, 2002, p. 33-72.

2. - VAN DEN DRIESSCHE, Bernard. «L'Antiquité et les moulages en plâtre en Belgique: 1830-1930 ». Dans TSINGARIDA, Athéna et VERBANCK-PIERARD, Annie (éd.). L'Antiquité au service de la Modernité ? La réception de l'antiquité classique en Belgique au XIX ${ }^{e}$ siècle. Bruxelles: Le Livre Timperman, 2008, p. 341-353.

3. - MONTENS, Valérie. «Cadre historique et institutionnel ». Dans LELOUP, Geert et MONTENS, Valérie. Archives institutionnelles des musées royaux d'Art et d'Histoire et archives des ASBL hébergées par l'institution. Tableau de tri des archives. Bruxelles: AGR, 2008, p. 27-50.

4. - MONTENS, Valérie. Les moulages des musées royaux d'Art et d'Histoire. Histoire de la collection et de l'atelier des reproductions en plâtre. Bruxelles, 2008.

5. - VAN KALCK, Michèle. «Le Musée des moulages ou 'collection des plâtres' ». Dans VAN KALCK, Michèle (éd.). Les Musées royaux des Beaux-Arts de Belgique. Deux siècles d'histoire, t. 1. Bruxelles: Racine, 2003, p. 261-264.

6. - Notice des tableaux et autres objets d'art exposés au Musée du Département de la Dyle, situé à Bruxelles, dans le local de la ci-devant Cour. Bruxelles, 1811, 64, nº114-115.

7. - Archives des musées royaux des Beaux-Arts (AMRBAB), dossier 235, note, s.d.

8. - VAN KALCK, Michèle, op.cit., t. 2, p. 683.

9. - AMRBAB, dossier 697, lettre du ministre à la commission administrative du musée, Bruxelles, 6 février 1860 .

10. - AMRBAB, dossier 697, copie d'une lettre de la commission administrative du musée au ministre, Bruxelles, 12 décembre 1860.

11. - Ibid.

12. - Instituée en 1835, la commission royale des Monuments était chargée de remettre des avis au ministre de l'Intérieur sur les réparations qu'exigent les monuments remarquables du pays et sur les plans relatifs aux constructions et réparations des édifices de culte et autres édifices publics. C'est à ce titre qu'elle intervint dans de nombreux dossiers relatifs aux musées.

13. - Archives des musées royaux des Beaux-Arts, dossier 697, lettre de la commission royale des Monuments au ministre, Bruxelles, 29 avril 1863.

14. - Reproduit par VAN KALCK, Michèle. "Le Musée des moulages ou "collection des plâtres" ». Dans VAN KALCK, Michèle (éd.). Les Musées royaux des Beaux-Arts de Belgique. Deux siècles d'histoire, t. 1. Bruxelles : Racine, 2003, p. 262.

15. - HYMANS, Henri. « Notice sur Jean-Baptiste Rousseau». Dans Biographie nationale, t. 20. Bruxelles, 1908-1910, col. 237-244.

16. - ROUSSEAU, Jean-Baptiste. "Le musée des plâtres au palais des Académies ». Bulletin des Commissions royales d'art et d'archéologie, 21, (1882), p. 279-280.

17. - Ibid., 312.

18. - Arrêté royal du 30 octobre 1884.

19. - Voir à ce propos LEBLANC, Claire. Des arts décoratifs aux arts industriels. Contribution à la genèse de l'Art nouveau en Belgique (1830-1893). Thèse de doctorat, U.L.B., 2005. 
20. - AMRBAB, dossier 2989, Lettre du ministre de l'Intérieur à la commission administrative du musée, 15 janvier 1884 .

21. - Ibid.

22. - MONTENS, Valérie. "Les "créateurs" du Musée du Cinquantenaire ». Bulletin des Musées royaux d'Art et d'Histoire. Bruxelles, t. 78, 2007, p. 121-136.

23. - Annales parlementaires, Chambre, 14 mai 1891, p. 1045.

24. - Annales parlementaires, Sénat, 22 février 1893, p. 140.

25. - Annales parlementaires, Chambre, 28 mai 1896, p. 1509.

26. - Archives des musées royaux d'Art et d'Histoire (AMRAH), archives de la commission royale belge des Échanges internationaux (CRBEI), dossier 22. Missions à l'étranger (secrétaire), Rapport sur les travaux du comité de la section artistique de la commission royale des Échanges internationaux pendant l'année 1898 (Extrait du Bulletin des Commissions royales d'art et d'archéologie, 1898).

27. - GRANGIER, Ophélie. L'Histoire matérielle des dix maquettes d'architecture en plâtre à fragments démontables créées sous la direction d'Anatole de Baudot pour l'Exposition universelle de 1900. Mémoire d'étude : Paris, École du Louvre, sous la dir. de Isabelle Cabillic, François Mirambet, 2013.

28. - Arch. MRAH, Arch. CRBEI, dossier 8. Procès-verbaux des séances 1871-1921.

\section{RÉSUMÉS}

Dès la fin des années 1830, la Belgique acquit des moulages réunis au «musée des Plâtres ». La création, en 1871, de la commission des Échanges internationaux permit le développement d'une nouvelle série. Ces deux collections furent rassemblées à la fin des années 1880 au sein des musées des Arts décoratifs et industriels pour former un panorama de l'histoire de la sculpture ancienne. $\mathrm{Au} \mathrm{xx}^{\mathrm{e}}$ siècle, priorité fut donnée aux moulages pouvant servir à l'enseignement des métiers d'art, ainsi qu'au patrimoine national. Après la Première Guerre mondiale, les moulages perdirent leur importance au sein des musées. Le démantèlement de la collection débuta au début des années 1930.

By the late 1830s, Belgium acquired plaster casts for its "Musée des plâtres". The creation in 1871 of the International Trade Commission allowed the development of a new series. Both collections were gathered in the late 1880s in the Museums of Decorative and Industrial Arts to form a panorama of the history of ancient sculpture. In the $20^{\text {th }}$ century, priority was given to plaster casts which could be used for the teaching of arts and crafts, and to national heritage. After the First World War, the plaster casts lost their importance in the museums. The dismantling of the collection began in the early 1930 s.

\section{INDEX}

Mots-clés : moulages, plâtres, reproductions, musée de Bruxelles, art monumental, Henry Rousseau, atelier de moulage

Keywords : plaster casts, plaster, monumental art 


\section{AUTEUR}

\section{VALÉRIE MONTENS}

Conservateur de la collection de céramiques (Europe), verres et vitraux aux Musées royaux d'art et d'histoire, Bruxelles v.montens@kmkg-mrah.be 